\title{
Occurrences, treatment and antibiotic resistant pattern of colibacillosis and salmonellosis in broiler
}

\author{
M. A. Hossain , M. R. Amin', M. D. I. Khan, M. L. Mollah ${ }^{b}$ and M. A. Amin \\ Dept. of Physiology and Pharmacology; \\ ${ }^{b}$ Dept. of Medicine, Surgery and Obstetrics, Faculty of Animal Science and Veterinary Medicine, \\ Patuakhali Science and Technology University (PSTU), Barisal, and \\ ${ }^{c}$ Central Disease Investigation Laboratory (CDIL), Dhaka, Bangladesh
}

\begin{abstract}
An experiment was conducted to investigate present occurrence of colibacillosis and salmonellosis in broiler and to find out the effective antibiotics for treatment of these diseases. The resistant pattern of Escherichia coli and Salmonella sp. against gentamicin, neomycin, oxytetracycline, amoxicillin, enrofloxacin and ciprofloxacin were also observed in Central Disease Investigation Laboratory (CDIL), Bangladesh. The occurrence of colibacillosis was $32.52 \%$ and salmonellosis was $13.17 \%$ but in mixed infection occurrence was $9.75 \%$ with colibacillosis and 2.92\% with salmonellosis. Neomycin (38.00\%) was mostly used for treatment of colibacillosis and enrofloxacin (25.93\%) was used for treatment of salmonellosis. Efficacy of neomycin and enrofloxacin was $92.10 \%$ in colibacillosis and $85.81 \%$ in salmonellosis respectively. The study also revealed that Escherichia coli were resistant against neomycin (38.88\%), oxytetracycline (50.55\%), amoxicillin (60.68\%), enrofloxacin (50.00\%) and ciprofloxacin (30.55\%). Salmonella sp. was resistant against neomycin (33.33\%), oxytetracycline (63.33\%), amoxicillin (63.33\%), enrofloxacin (52.38\%) and ciprofloxacin (52.38\%) respectively. Gentamicin (100\%) was highly sensitive for the treatment of colibacillosis and salmonellosis.
\end{abstract}

Keywords: Salmonellosis, colibacillosis, occurrences, treatments and antibiotic resistant

Please cite this article as: Hossain, M. A., Amin, M. R., Khan, M. D. I., Mollah, M. L. \& Amin, M. A. (2015). Occurrences, treatment and antibiotic resistant pattern of colibacillosis and salmonellosis in broiler. Journal of Bioscience and Agriculture Research 04(02): 67-73.

This article is distributed under terms of a Creative Common Attribution 4.0 International License

\section{Introduction}

Poultry products were one of the most important protein sources for human beings throughout the World. A major portion (44\%) of the population of Bangladesh lives below absolute poverty line and the number of landless poor has been increasing by $3.4 \%$ per annum (BBS, 2000). About $47.5 \%$ people receive less than 1900 calorie per person per day as against the standard 2300 calories. Avian colibacillosis and salmonellosis has been found to be major infectious diseases of all ages of broiler. These diseases occur sporadically or enzootically in most of the countries of the world including Bangladesh. The most important reservoir of Escherichia coli was the intestinal tract of animals, 
including poultry. In chickens, there were about 109 colony-forming units (CFU) of bacteria per gram of feces. Of these, $106 \mathrm{CFU}$ were Escherichia coli, 10-15\% of which were pathogenic serogroups and probably infect most mammals and birds thus having a cosmopolitan distribution. Coliforms transmitted between poultry and humans (Ojeniyi, 1989). Egg transmission of pathogenic Escherichia coli was common and responsible for high chicken mortality. Pathogenic coliforms were more frequent in the gut of the newly hatched chicks than in eggs from which they hatched (Harry and Hemsly, 1965). Salmonellosis was a serious systemic disease of domestic poultry which cause large scale economic losses (Shivaprasad et al.1997). It causes severe economic losses of the poultry with morbidity and mortality varying in chicken from $10-50 \%$ or more (Vaillancourt et al., 1992). Salmonellosis was distributed in many countries of the world, and has economic significance (Barrow et al., 1992). The major of economic loss results from mortality and decrease productivity in affected birds (Otaki, 1995). Antimicrobial resistance, the ability of microorganisms (notably bacteria) to withstand antimicrobial agents (antibiotics), was an important and growing public health issue. However, over the years bacteria that were once controlled by these drugs have developed resistance so that common infections in humans can cause significant harm and even death. Escherichia coli was the primary causative agent of cellulitis, septicemia, and air sacculitis in poultry and Salmonella sp. was the causative agent of pullorum disease, Fowl typhoid and Fowl paratyphoid (Gomis et al., 1997). Therefore, these were the most significant poultry bacterial pathogen. There were several antimicrobials that have been approved for treatment of Escherichia coli and Salmonella sp. infections in broiler. However, some of these antimicrobials were not cost-effective, while others were ineffective due to acquired resistance (Bass et al., 1999). The long-term use of antimicrobials for therapy and growth promotion in animals selects for drug resistance in gram-negative pathogens (Levy et al., 1976). Until today very little works have been performed in our country on antibiotic resistant pattern in broiler. Considering all these constraints, this pioneering work has been under taken in this country to determine the present occurrence of colibacillosis and salmonellosis in broiler and find out the most effective antibiotics for treatment of these diseases in Kapasia upazilla of Gazipur district. The resistant pattern of Escherichia coli and Salmonella sp. against antibiotics (gentamicin, neomycin, oxytetracycline, amoxicillin, enrofloxacin and ciprofloxacin) were also observed in the study.

\section{Materials and Methods}

The experiment was conducted at Kapasia upazilla of Gazipur district in Bangladesh during 23rd March, 2014 to $14^{\text {th }}$ May, 2014. Antibiotic resistant pattern in broiler were observed in Central Disease Investigation Laboratory (CDIL), Bangladesh. 615 birds from different farms were subjected to postmortem examination. From them 36 isolates of Escherichia coli and 21 isolates of Salmonella sp. prepared from single colibacillosis and salmonellosis cases for Cultural Sensitivity (CS) Test. Diagnosis of disease was made on the basis of history, signs and symptoms prior to death of birds, post-mortem lesions of dead birds by post-mortem examination, supported by microbiological examination when indicated, using standard methods (culture at MacConkey agar and SS agar) for bacterial identification. The post-mortem examinations were performed and lesions were recorded as early as possible after death of the birds. Treatment was given according to presumptive diagnosis. Study the type of antibiotics prescribed against those clinical cases. Both Escherichia coli and Salmonella sp. were gram negative bacteria and were used gram negative bacteria sensitive antibiotics or broad spectrum antibiotics. Gentamicin, neomycin, oxytetracycline, amoxicillin, enrofloxacin and ciprofloxacin were used for the treatment of the colibacillosis and salmonellosis.

Cases suspected for colibacillosis and salmonellosis after post-mortem examination were subjected to microbial examination (Collins and Lyne, 1976). Liver sample was considered for microbial examination. $1 \mathrm{gm}$ of liver samples was taken and kept in the selinite broth. Then tubes of selinite broth with liver were incubated for overnight at $37^{\circ} \mathrm{C}$. In case of immediate use after few hours, sample was taken by sterilized cotton swab just after searing. There were many laboratory examinations and techniques for confirm diagnosis of colibacillosis and salmonellosis such as isolation and identification of the organisms, serological test, culturing on various selective media and observation of colony characteristics and also observation of the organisms under microscope after staining. For suspected cases of colibacillosis inoculation from both broth sample and swab sample culturing were done at MacConkey agar and blood agar plate and were kept for overnight incubation 
and observed. Both lactose fermenting and non-lactose fermenting colonies were found. More confirmation was made by biochemical tests of pink colored colonies of Escherichia coli. Various biochemical tests were done for identification (Cruickshank et al., 1995). Notable performed biochemical tests were SIM (Sulfied Indole Motility Test), TSI (Triple Sugar Iron) agar stab and Urea. Among all confirmatory diagnostic methods culturing on selective media (MacConkey Agar and SS Agar) and observation of colony characteristics as well as Biochemical Test at TSI agar stab were selected for salmonellosis.

After confirmation the isolates as Escherichia coli and Salmonella sp. were subjected for antibiotic susceptibility tests. Upon Escherichia coli and Salmonella sp. identification, antibiotic sensitivity test were performed on the isolates by standard technique of EUCAST and recommendation done by the guidelines of National Committee for Clinical Laboratory Standards (NCCLS, 1997). Antibiotics selected for susceptibility testing included a panel of antimicrobial agents of interest to the poultry industry and human public health authorities. 6 drugs were selected on the basis of their range of activity against enterobacteria and on their use in the poultry farming. The following antibiotics and disc potencies were used: GN: gentamicin $(120 \mu \mathrm{g})$, NEO: neomycin $(30 \mu \mathrm{g})$, CIP: ciprofloxacin $(5 \mu \mathrm{g})$,

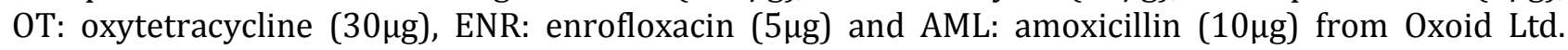
(Basingstoke, Hampshire, England). The antibiotic susceptibility tests were performed in MullerHinton agar (Difco) by EUCAST micro-disc diffusion techniques. Measurement of the growth inhibition zone permitted the classification of each isolates as susceptible, intermediate and resistant according to data collected from Bacteriology Laboratory of CDIL (Central Disease Investigation Laboratory). The result of antibiotic sensitivity test was then recorded and analyzed.

\section{Results and Discussion}

\section{Total number of diseases}

An infectious disease was any disease caused by invasion of a host by a pathogen which subsequently grows and multiplies in the body. Infectious diseases were often contagious, which means they can be spread directly or indirectly from one living thing to another. Diseases (bacterial, viral, fungal etc.) are diagnosed by post-mortem examination includes colibacillosis, salmonellosis, necrotic enteritis, fowl cholera, mycoplasmosis, avian streptococcosis, avian influenza, fowl pox, infectious bursal disease, Newcastle disease, coccidiosis etc.

Table 01. List of diseases available in the study area

\begin{tabular}{|c|c|c|c|}
\hline Case types & Name of the diseases & No. of birds affected & Frequency (\%) \\
\hline \multirow{10}{*}{$\begin{array}{l}\text { Single } \\
\text { Infections }\end{array}$} & Colibacillosis & 200 & $32.52 \%$ \\
\hline & Salmonellosis & 81 & $13.17 \%$ \\
\hline & Necrotic Enteritis & 59 & $9.59 \%$ \\
\hline & Avian Streptococcosis & 5 & $0.81 \%$ \\
\hline & Mycoplasmosis/CRD & 23 & $3.74 \%$ \\
\hline & Coccidiosis & 34 & $5.53 \%$ \\
\hline & Aspergillosis & 15 & $2.44 \%$ \\
\hline & Infectious Bursal Disease & 73 & $11.87 \%$ \\
\hline & Newcastle disease & 37 & $6.02 \%$ \\
\hline & Fowl pox & 4 & $0.65 \%$ \\
\hline \multirow{6}{*}{$\begin{array}{c}\text { Mixed } \\
\text { Infections }\end{array}$} & Colibacillosis+Avian streptococcosis & 3 & $0.49 \%$ \\
\hline & Colibacillosis+Newcastle disease & 11 & $1.79 \%$ \\
\hline & Colibacillosis+Coccidiosis & 6 & $0.98 \%$ \\
\hline & Colibacillosis+Infectious Bursal Disease & 12 & $1.95 \%$ \\
\hline & Colibacillosis+Mycoplasmosis & 10 & $1.62 \%$ \\
\hline & Colibacillosis+Salmonellosis & 18 & $2.92 \%$ \\
\hline \multirow{3}{*}{ Others } & Mycotoxicosis & 7 & $1.14 \%$ \\
\hline & Malnurtition & 7 & $1.14 \%$ \\
\hline & Stress & 10 & $1.62 \%$ \\
\hline & Total & 615 & $100 \%$ \\
\hline
\end{tabular}


Some conditions were caused by a disturbance of normal metabolic functions either through inadequate or inappropriate nutrition or impaired nutrient utilization (Jordan and Pattison, 1996). List of different diseases and their frequency in the study area were shown in table 01.

The diagnosed diseases included colibacillosis (32.52\%), salmonellosis (13.17\%), necrotic enteritis (9.59\%), avian streptococcosis (0.81\%), mycoplasmosis / CRD (3.74\%), coccidiosis (5.53\%), aspergillosis (2.44\%), infectious bursal disease (11.87\%), Newcastle disease $(6.02 \%)$ and fowl pox $(0.65 \%)$ as single infections. Mixed infections were available as colibacillosis + avian streptococcosis $(0.49 \%)$, colibacillosis + Newcastle disease $(1.79 \%)$, colibacillosis + coccidiosis $(0.98 \%)$, colibacillosis + infectious bursal disease (1.95\%), colibacillosis + mycoplasmosis $(1.62 \%)$ and colibacillosis + salmonellosis (2.92\%). Non-infectious cases were mycotoxicosis $(1.14 \%)$, malnurtition $(1.14 \%)$ and stress $(1.62 \%)$.

\section{Occurrences of colibacillosis and salmonellosis}

Among the diseases in commercial broiler farms, the occurrences rate of death due to colibacillosis was the highest. Therefore the occurrences is $32.52 \%$ for single colibacillosis case but in case of mixed colibacillosis the occurrences was $9.75 \%$ and mixed salmonellosis occurrences was $2.92 \%$. The occurrence of single salmonellosis was 13.17\%. Similarly, Bhattacharjee and Majumder (2001) diagnosed the occurrences of avian colibacillosis was highest among the bacterial diseases in chicken at Central Disease Investigation Laboratory (CDIL), Dhaka.

\section{Usage and efficacy of antibiotics for treatment of colibacillosis and salmonellosis}

Antibiotics were extremely important tool in the efficient production such as meat and eggs. At subtherapeutic levels in diets, antibiotics improve growth rate and efficiency of feed utilization, reduce mortality and morbidity and improve reproductive performance. At high levels antibiotics help to prevent disease in exposed animals and to treat diseases (Cromwell, 1999). For the treatment of colibacillosis, gentamicin (18.00\%), neomycin (38.00\%), oxytetracycline (8.00\%), amoxicillin (5.00\%), enrofloxacin $(15.00 \%)$ and ciprofloxacin $(16.00 \%)$ were used in that area. Gentamicin showed (100\%) effective for the treatment of colibacillosis where neomycin was $92.10 \%$, oxytetracycline $62.50 \%$, amoxicillin 50\%, enrofloxacin $73.33 \%$ and ciprofloxacin $65.62 \%$ effective. For the treatment of salmonellosis gentamicin (22.22\%), neomycin (17.28\%), oxytetracycline (11.11\%), amoxicillin (6.17\%), enrofloxacin (25.93\%) and ciprofloxacin (17.28\%) were used in that area. In the treatment of salmonellosis gentamicin showed $100 \%$ effective followed neomycin (71.42\%), oxytetracycline (55.56\%), amoxicillin (60\%), enrofloxacin (85.81\%) and ciprofloxacin (64.29\%).

\section{Comparison of antimicrobial resistant patterns of Escherichia coli and Salmonella sp.}

Antibiotic resistant is a form of drug resistance whereby some sub-populations of a microorganism, usually a bacterial species, were able to survive after exposure to one or more antibiotics. Antibiotic resistant is a serious and growing phenomenon in contemporary medicine and has emerged as one of the pre-eminent public health concerns of the $21^{\text {st }}$ century. When bacteria change so antibiotics no longer work in people who need them to treat infections is now a major threat to public health.

Table 02. Sensitivity pattern of Escherichia coli

\begin{tabular}{l|cccc}
\hline \multirow{2}{*}{$\begin{array}{c}\text { Name of the } \\
\text { antibiotics }\end{array}$} & \multirow{2}{*}{$\begin{array}{c}\text { No. of isolates } \\
\text { tested }\end{array}$} & Resistant (\%) & Susceptible (\%) & Intermediate (\%) \\
\cline { 3 - 5 } & 36 & 0 & 100 & 0 \\
\hline Gentamicin & 36 & 38.88 & 61.12 & 0 \\
Neomycin & 36 & 50.55 & 19.45 & 30 \\
Oxytetracycline & 36 & 60.88 & 11.12 & 28 \\
Amoxicillin & 36 & 50 & 50 & 0 \\
Enrofloxacin & 36 & 30.55 & 69.45 & 0 \\
Ciprofloxacin & & & & \\
\hline
\end{tabular}


Antibiotics (gentamicin, neomycin, oxytetracycline, amoxicillin, enrofloxacin and ciprofloxacin) were chosen in the study as these antibiotics using in both veterinary and human medical practices. Escherichia coli isolates were tested for resistant towards 6 above mentioned antibiotics (Table 2). Resistances were commonly observed against neomycin (38.88\%), oxytetracycline (50.55\%), amoxicillin (60.88\%), enrofloxacin (50\%) and ciprofloxacin (30.55\%). But high levels of sensitivity were found against gentamicin (100\%) and ciprofloxacin (69.45\%). Those above-mentioned isolates had considerable sensitivity to neomycin (61.12\%). But in previous study all the poultry Escherichia coli isolates were found resistant to tetracycline (Bauer et al., 1966). High level of resistance against tetracycline because most of the farmers used commercially available tetracycline in the poultry feed regularly. The farmers used antibiotics largely for three purposes in poultry farms: therapeutic use to treat sick flock; prophylactic use to prevent infections in the flock; as growth promoters to improve feed utilization and production (Barton, 2000). Most of the environmental strains were sensitive to ciprofloxacin (76\%), and gentamicin (97\%) (Alam et al., 2006). Poultry isolates 72\% Escherichia coli were found sensitive to gentamicin (Biswas et al., 2001). So, the results of ciprofloxacin resistance and gentamicin sensitivity in poultry isolates were mostly similar with environmental Escherichia coli. In another study, a high frequency of resistance to tetracycline, kanamycin, neomycin, cephalothin, streptomycin, and erythromycin was observed. Many strains were resistant to several antibiotics, but no pattern predominates (Allan et al., 1993). Amoxicillin is a broad-spectrum antibiotic of penicillin group. But highest resistance found against these antibiotics $60.88 \%$. No data is still available about the high level of resistance against amoxicillin of Escherichia coli isolates in Bangladesh. Enrofloxacin and ciprofloxacin both were belong to $2^{\text {nd }}$ generation fluroquinolones. But variable resistance pattern found Escherichia coli isolates against this group and it is highest in enrofloxacin $50 \%$. It is due to more use of enrofloxacin than ciprofloxacin in poultry.

Table 03. Sensitivity pattern of Salmonella sp.

\begin{tabular}{l|cccc}
\hline Name of the & No. of isolates & \multicolumn{3}{c}{ Sensitivity of pattern } \\
\cline { 3 - 5 } antibiotics & tested & Resistant (\%) & Susceptible (\%) & Intermediate (\%) \\
\hline Gentamicin & 21 & 0 & 100 & 0 \\
Neomycin & 21 & 33.33 & 66.67 & 0 \\
Oxytetracycline & 21 & 63.33 & 16.67 & 20 \\
Amoxicillin & 21 & 63.33 & 16.67 & 20 \\
Enrofloxacin & 21 & 52.38 & 47.62 & 0 \\
Ciprofloxacin & 21 & 52.38 & 47.62 & 0 \\
\hline
\end{tabular}

Salmonella sp. isolates were tested for resistant towards 6 above mentioned antibiotics (Table 3). Resistances of Salmonella sp. isolates were commonly observed against neomycin (33.33\%), oxytetracycline (63.33\%), amoxicillin (63.33\%), enrofloxacin (52.38\%) and ciprofloxacin (52.38\%). But high levels of sensitivity were found against gentamicin (100\%) and neomycin (66.67\%). Similar findings were also reported by Molla et al. (2003). Gentamicin is exceptional aminoglycosidic antibiotic which is bacteriocidal for negative bacteria. Salmonella sp. isolates were most sensitive to gentamicin among all antibiotics. Highest resistance of Salmonella sp. isolates is found $63.33 \%$ in case of both oxytetracycline and amoxicillin. Highest resistance found against amoxicillin for both Escherichia coli and Salmonella sp. isolates is great threat to poultry industry. Intermediate level of resistance found $52.38 \%$ in both enrofloxacin and ciprofloxacin of $2^{\text {nd }}$ generation fluroquinolones group. In the present study all Salmonella sp. and Escherichia coli isolates were susceptible to gentamicin. This may be explained by the limited use and high cost of this antimicrobial that would reduce their frequent utilization both in the veterinary and public health practices in Kapasia area. Poultry farmers avoid gentamicin for being fear of production loss. Further detailed epidemiological and molecular studies are essential on the frequency, sources of acquisition of resistant genes and distribution of antimicrobial resistant of Escherichia coli and Salmonella sp. in broiler. Antibiotic use should be controlled by the authority to prevent the chance of antibiotic resistance in broiler. Antibiotic resistance monitoring program should also be establish in near future to prevent the global antibiotic resistance problem. 


\section{Conclusion}

Colibacillosis and salmonellosis found as the potential importance disease for broiler because their occurrences were high in Kapasia upazilla of Gazipur district of Bangladesh. Gentamicin, neomycin, oxytetracycline, amoxicillin, enrofloxacin and ciprofloxacin were commonly used for the treatment of colibacillosis and salmonellosis. Colibacillosis and salmonellosis gradually increase resistant due to indiscriminate use of antibiotics in feed and water. Further detailed epidemiological and molecular studies are essential on the frequency, sources of acquisition of resistant genes and distribution of antimicrobial resistant of Escherichia coli and Salmonella sp. in broiler. Antibiotic use should be controlled by the authority to prevent the chance of antibiotic resistance in broiler. Antibiotic resistance monitoring program should also be establish in near future to prevent the global antibiotic resistance problem.

\section{Acknowledgement}

The support of Upazilla Livestock Development Centre, Kapasia, Gazipur and Central Disease Investigation Laboratory (CDIL), Bangladesh for conducting the experiment is gratefully acknowledged.

\section{References}

[1]. Alam, M., Ahasan, S., Pazhani, G.P., Tamura, K., Ramamurthy, T., Gomes, D.J. \& Nair, B. (2006). Phenotypic and molecular characteristics of Escherichia coli isolated from aquatic environment of Bangladesh. Microbiology and Immunology 50(5), 359-370.

[2]. Allan, B. J., Hurk, J. V. \& Potter, A. A. (1993). Characterization of Escherichia coli isolated from cases of avian colibacillosis, Canadian Journal of Veterinary Research 57(3), 146-151.

[3]. Barrow, T. M., Umapathy, R., Srinivasa, S. R., Gnaprakasam, V. and Nagarajan, V. (1992). Agricultural use of antibiotics and the evolution and transfer of antibiotics $\left(2^{\text {nd }}\right.$ ed.). Sander press. pp 35-38.

[4]. Barton, M. D. (2000). Antibiotic use in animal feed and its impact on human health. Nutrition research reviews 13, 279-299. [ doi: 10.1079/095442200108729106]

[5]. Bass, L. C. A., Liebert, M., Lee, M. D., White D. G, Summers, A. O., Thayer, S. G. \& Maurer, J. J. (1999). Occurrences and characterization of integrants, genetic elements mediating multiple-drug resistance in avian E. coli. Antimicrobial Agent and Chemotherapy 43, 2925-2929.

[6]. Bauer, A. W., Kirby, W. M., Sheris, J. C. \& Truck, M. (1966). Antibiotic susceptibility testing by a standardized single disk method. Technical bulletin of the Registry of Medical Technologists 36(3), 49-52.

[7]. BBS, (2000). Agriculture sample survey of Bangladesh. Planning Division, Ministry of Planning, Government of peoples Republic of Bangladesh. Dhaka. 1: 34-35.

[8]. Bhattacharjee, A. \& Majumder, P. (2001). Fowl Typhoid outbreak in broiler chick flocks in Tripura and its control. The Indian Journal of Animal Sciences 71 (11), 1034-1035.

[9]. Biswas, P. K., Faruque, R., Ahmed, S. \& Dey, V. C. (2001). Antibiotic sensitivity pattern of pathogenic Escherichia coli isolated from fayoumi chicken. Bangladesh Journal of Microbiology 18(3), 121-126.

[10]. Collins, C. H. \& Lyne, P. M. (1976). Microbiological methods (4th ed.). Buttenworths press, London. pp 275-278.

[11]. Cromwell, G. L. (1999). Country report on the management and health problems of rural poultry stock in Nigeria. In: Proceedings of Centre for Tropical Agriculture. pp 175-182.

[12]. Cruickshank, R., Duguid, B. P., Marimion, J. H. \& Swain, R. H. A. (1995). Medical Microbiology (12 ${ }^{\text {th }}$ ed.). Churchill Livingstone, London. pp 126-127.

[13]. Gomis, S. M., Goodhope, L. R., Kumor, N. H., Caddy, C. A., Riddell, A. A., Petter, R. \& Allan, J. J. (1997). Experimental reproduction of Escherichia coli, cellulitis and septicemia in broiler chickens. Avian Diseases 41, 234-240. 
[14]. Harry, E. G. \& Hemsley, L. A. (1965). The relationship between environmental contamination with septicemia strains of E. coli and their occurrences in chickens. The Veterinary Record 77, 241-245.

[15]. Jordan, F. T. W. \& Pattison, M. (1996). Poultry Diseases (4th ed.). W.B. Saunders Company Ltd., London NW1 7DX.

[16]. Levy, S. B., FitzGerald, G. B. \& Macone, A. B. (1976). Spread of antibiotic-resistant plasmids from chicken to chicken and from chicken to man (4th ed.). Blackwell Publication, London. $p 361$.

[17]. Molla, B., Mesfin, A. \& Alemayehu, D. (2003). Multiple antimicrobial-resistant salmonella serotypes isolated from chicken carcasses, Ethiopia. Ethiopian Journal of Health Development 17(2), 131-149.

[18]. NCCLS (1997). Performance standards for antimicrobial disk susceptibility tests. Approved standard. pp 543-555.

[19]. Ojeniyi, A. A. (1989). Direct transmission of Escherichia coli from poultry to humans. Epidemiology and Infection 103, 513-522.

[20]. Otaki, Y. (1995). Poultry disease control programme in Japan. Asian Livestock 20, 65-67.

[21]. Shivaprasad, H. L., Calnek, B. W., Barnes, H. J., Bearb, C. W., McDoughald, L. R. \& Saif, Y. M., (1997). Diseases of Poultry (10 th ed.). Iowa State University Press. pp 82-96.

[22]. Vaillancourt, J. P., Elfadil, A. \& Bissailon, J. R. (1992). Cellulitis in poultry, Canada Poultryman 79, 34-37. 